\title{
Anticholinergic discontinuation and cognitive functions in patients with schizophrenia: a pharmacist-physician collaboration in the outpatient department
}

This article was published in the following Dove Press journal: Integrated Pharmacy Research and Practice

\section{Thanompong}

Sathienluckana'

Weerapon Unaharassamee ${ }^{2}$

Chuthamanee Suthisisang ${ }^{3}$

Orabhorn Suanchang ${ }^{4}$

Thanarat Suansanae ${ }^{5}$

'Faculty of Pharmacy, Siam

University, Bangkok, Thailand;

${ }^{2}$ Department of Psychiatry, Somdet

Chaopraya Institute of Psychiatry,

Bangkok, Thailand; ${ }^{3}$ Department of

Pharmacology, Faculty of Pharmacy,

Mahidol University, Bangkok, Thailand;

${ }^{4}$ Department of Pharmacy, Somdet

Chaophraya Institute of Psychiatry,

Bangkok, Thailand; ${ }^{5}$ Department

of Pharmacy, Faculty of Pharmacy,

Mahidol University, Bangkok, Thailand
Introduction: Cognitive impairment is a core feature and shows the highest impact on functional outcome in patients with schizophrenia. There have been no previous studies investigating the role of the pharmacist in a multidisciplinary team on cognitive outcomes in patients with schizophrenia.

Purpose: We evaluated the impact of pharmacist intervention on cognitive outcomes in patients with schizophrenia by focusing on anticholinergic discontinuation.

Patients and methods: A prospective, open-label, randomized, controlled study was conducted. Patients with schizophrenia were randomly assigned to either the pharmacist intervention or usual care groups. In the pharmacist intervention group, the pharmacist identified drug-related problems (DRPs) and provided a pharmacotherapy suggestion, while there was no intervention in the usual care group. The primary outcome was mean change from baseline of executive function by using Wisconsin Card Sorting Test (WCST) perseverative errors within the pharmacist intervention group at week 12 .

Results: A total of 30 patients completed the study (13 in the pharmacist intervention group and 17 in the usual care group). WCST perseverative errors at the end of the study within the pharmacist intervention group improved significantly from baseline $(P=0.003)$. DRPs at week 12 were reduced by $85.19 \%$ and $9.76 \%$ in the pharmacist intervention and usual care groups, respectively. The most common intervention was the discontinuation of anticholinergics in patients without extrapyramidal side effects.

Conclusion: Added-on pharmacist intervention in a multidisciplinary team could help to improve cognitive functions in patients with schizophrenia by reducing DRPs and optimizing the drug therapy regimen, especially for anticholinergic discontinuation.

Keywords: pharmacist intervention, cognitive functions, schizophrenia, anticholinergic

\section{Introduction}

Schizophrenia is a chronic psychiatric disorder that comprises heterogeneous conditions including positive, negative, cognitive, and affective symptoms. ${ }^{1}$ These symptoms can impair many aspects of the functional outcomes that are social and vocational functioning. Positive and negative symptoms can be minimized by currently available treatment options, while there are limited strategies to improve cognitive functions in patients with schizophrenia. ${ }^{1}$ Cognitive impairment is a core feature of schizophrenia that can be found prior to the development of first psychotic episode and continues throughout the illness. ${ }^{2,3}$ In addition, cognitive impairment shows the highest impact on
Correspondence: Thanarat Suansanae Department of Pharmacy, Faculty of Pharmacy, Mahidol University, 447 SriAyuthaya Road, Ratchathewi, Bangkok 10400, Thailand

Tel +66 26448694

Fax +66 26448694

Email thanarat.sua@mahidol.ac.th 
functional outcomes and is highly associated with relapse. ${ }^{4-7}$ Thus, improvement of cognitive functions is one of the key components of disease recovery. The Measurement and Treatment Research to Improve Cognition in Schizophrenia (MATRICS), a project under the National Institute of Mental Health, reported that the key cognitive domains in schizophrenia are attention/vigilance, speed of processing, verbal learning and memory, visual learning and memory, working memory, reasoning and problem solving (executive function), and social cognition. ${ }^{2}$ Verbal learning and memory, attention, and executive function are the most commonly impaired domains in patients with schizophrenia. ${ }^{2,4}$ Of these domains, executive function was strongly associated with community functioning and skill acquisition. ${ }^{1}$

Current strategies to improve cognitive functions in schizophrenia comprise non-pharmacological and pharmacological treatments. The main pharmacological intervention is the avoidance or discontinuation of medication, especially anticholinergic drugs, which can impair cognitive functions that are unnecessarily long-term used in patients with schizophrenia. Other pharmacological interventions include the selection of an appropriate antipsychotic agent, which exhibits benefits either for negative symptoms or cognitive functions, and adjunctive treatment with some antidepressants. ${ }^{8}$ However, the success of these strategies can prove difficult if there is no collaboration between the clinical pharmacist and physician in the health care team.

Pharmacist intervention by delivering pharmaceutical care in a multidisciplinary care team has been shown to improve clinical outcomes in a variety of patients with chronic diseases such as heart failure, hypertension, and diabetes. ${ }^{9-11}$ As concerns psychiatric disorders, some clinical studies have addressed pharmaceutical care in psychiatric patients with depression and autism spectrum disorder. ${ }^{12,13}$ The results also revealed that pharmacist intervention could help to improve clinical outcomes in these patients. Regarding schizophrenia, even though a high frequency of drugrelated problems (DRPs) has been reported, especially in the use of long-term anticholinergic drugs, ${ }^{14,15}$ research on the pharmacist's role with these patients is still limited. A few studies demonstrated that pharmacist intervention could help to improve psychopathological symptoms more than usual care. ${ }^{14}$ However, cognitive outcome has not been previously investigated. Therefore, the objective of our study was to evaluate the impact of pharmacist intervention on cognitive functions in patients with schizophrenia by focusing on anticholinergic discontinuation.

\section{Materials and methods}

\section{Setting}

This was a 12-week, prospective, open-label, randomized, controlled study designed to evaluate the effects of pharmacist intervention on cognitive functions in patients with schizophrenia. The study was conducted at the outpatient department of Somdet Chaopraya Institute of Psychiatry, Bangkok, Thailand. Eligible patients were randomized into pharmacist intervention and usual care groups by simple randomization. Real-life care by psychiatrists in the outpatient department without pharmacist involvement was selected as the usual care group. The study was approved by the ethics medical committee of the Somdet Chaopraya Institute of Psychiatry, and all patients had to sign an informed consent prior to entering the study.

\section{Participants}

Patients who visited the outpatient department of Somdet Chaopraya Institute of Psychiatry from February 2015 to June 2015 were recruited by a clinical pharmacist. The inclusion criteria were as follows: 1) aged between 18 and 50 years; 2) had been diagnosed with schizophrenia according to the Diagnostic and Statistical Manual of Mental Disorders, fourth edition (DSM-IV); 3) had received stable agent and dosage regimen of antipsychotics for at least 2 months, which was an adequate duration to assess the therapeutic effect of medications; 4) had mild to moderate symptoms of schizophrenia as determined by the Brief Psychiatric Rating Scale (BPRS) of 32-54; and 5) had schizophrenia after first diagnosis for a duration of less than 10 years. Patients were excluded if they had mental retardation, delirium, or had communication impairment, which affected their ability to perform cognitive function tests.

Concerning the characteristics of participants, because this study focused on cognitive functions, only patients with stable and mild to moderate symptom severity of schizophrenia were recruited. If patients had severe or unstable psychotic symptoms, the treatment goal might be distorted to focus on the management of positive symptoms instead of cognitive symptoms. Patients who had the illness for a duration of less than 10 years were recruited because most studies found that improvement in the executive function is seen in the early phase of schizophrenia; however, there is no improvement if the duration of illness is longer than 10 years. ${ }^{16-18}$

\section{Pharmacist interventions}

At baseline, all participants underwent cognitive function tests by clinical psychologists. However, symptom severity, 
medication history review, medication adherence, and identification of DRPs were conducted by the clinical pharmacist before the patients met psychiatrists.

In the pharmacist intervention group, a clinical pharmacist undertook the responsibility to provide pharmaceutical care to all patients. These included conducting a treatment plan that suggested treatment strategies to psychiatrists, which could help improve the cognitive functions in their individual patients (Figure 1). These strategies included the following. 1) Avoiding or discontinuing medications that interfere with cognitive functions, especially the use of anticholinergic drugs in patients who had no extrapyramidal side effects (EPS) from antipsychotics for at least 4 weeks and no history of severe EPS. ${ }^{8,19,20}$ Severe EPS is defined as life-threatening adverse effects or adverse effects that significantly interfere with the activity of daily living of patients. Schedule for the tapering off anticholinergic drug was a decreased dose of about 2-5 mg of trihexyphenidyl equivalent every 6 weeks. 2) Suggesting to add antidepressants or substitute second-generation antipsychotics (SGAs) for first-generation antipsychotics (FGAs) in patients who still exhibited predominant negative symptoms. ${ }^{21-26} 3$ ) Considering the substitution of SGAs for FGAs in patients identified as having cognitive impairment based on cognitive function tests and with stable psychotic symptoms. ${ }^{16,27}$ 4) Decreasing the dose of antipsychotics in patients with EPS but whose other psychotic symptoms were stable. ${ }^{28}$

\section{Clinical assessment}

The cognitive tests were performed at baseline and at week 12 by the same clinical psychologists who were blinded to group assignment. These assessments focused on the measures of executive function, verbal learning and memory, and speed of processing. These comprised five tests each examining different cognitive domains as shown in Table 1 (for details of each cognitive tests, refer Table S1).

Psychopathological symptoms were rated with BPRS by a clinical pharmacist who had been trained in BPRS assessment. BPRS was performed at baseline and repeated at weeks 6 and 12. DRPs were identified and categorized into seven categories according to Cipolle/Morley/Strand classification. ${ }^{29}$ Adherence was measured by multiple methods including pill count, patient report, and caregiver report. Regarding the patient report and caregiver report, visual analogue rating scale (VAS) was used to assess nonadherence by estimating the percentage of medication dose that patients had taken as prescribed $(0 \%-100 \%)$. Nonadherence was defined as following less than $80 \%$ of the prescribed medication. If the data conflicted between methods, the pill count method was used, which is an objective measurement, as the first decision.

\section{Study outcomes}

The primary outcome was the mean change from baseline of Wisconsin Card Sorting Test (WCST) perseverative errors within the pharmacist intervention group at week 12 . The secondary outcomes comprised: 1) mean change from baseline to week 12 of cognitive tests (WCST, Wechsler Memory Scale; WMS, Stroop Color Word Test, Trail Making Test A [TMT A], Trail Making Test B [TMT B]) between pharmacist intervention and usual care groups; 2) mean change from baseline to week 12 of BPRS between pharmacist intervention and usual care groups; 3 ) proportion of patients who had a clinical response of psychopathological symptoms (defined as patients who had BPRS at the end of study decreasing at least 30\% from baseline) between pharmacist intervention and usual care groups; and 4) frequencies of identified DRPs at baseline and week 12 between pharmacist intervention and usual care groups.

\section{Statistical analyses}

Regarding the sample size, there was no clinical study comparing the effect between pharmacist intervention and usual care in patients with schizophrenia, which made it difficult to set the sample size for determining the differences in changes in WCST perseverative errors between groups. Therefore, mean change from baseline of WCST perseverative error within the pharmacist intervention group was used as the primary outcome of this study. Sample size was calculated based on Purdon et $\mathrm{al}^{16}$ which revealed that treatment with SGAs could improve WCST perseverative errors approximately 7.25 points from baseline and relatively used anticholinergic drugs less than FGA, could improve WCST perseverative errors approximately 7.25 points at end point when compared from baseline. Therefore, a minimum total of 39 patients needed to be recruited for a significance level of $\alpha=0.05$ and for a power of at least $80 \%(\beta<0.20)$.

Baseline characteristics were compared between groups using Fisher's exact test for nominal data and Mann-Whitney $U$ test for continuous data. The primary analytical approach was per-protocol analysis. Mean change in WCST perseverative errors within the pharmacist intervention group at week 12 was tested by the Wilcoxon signed-rank test. Mean change from baseline of the cognitive tests and BPRS between groups were tested by the Mann-Whitney $U$ test. Proportion of patients who achieved response between groups was compared using the Fisher's exact test. Descriptive analysis was 
Assessed for eligibility $(n=128)$

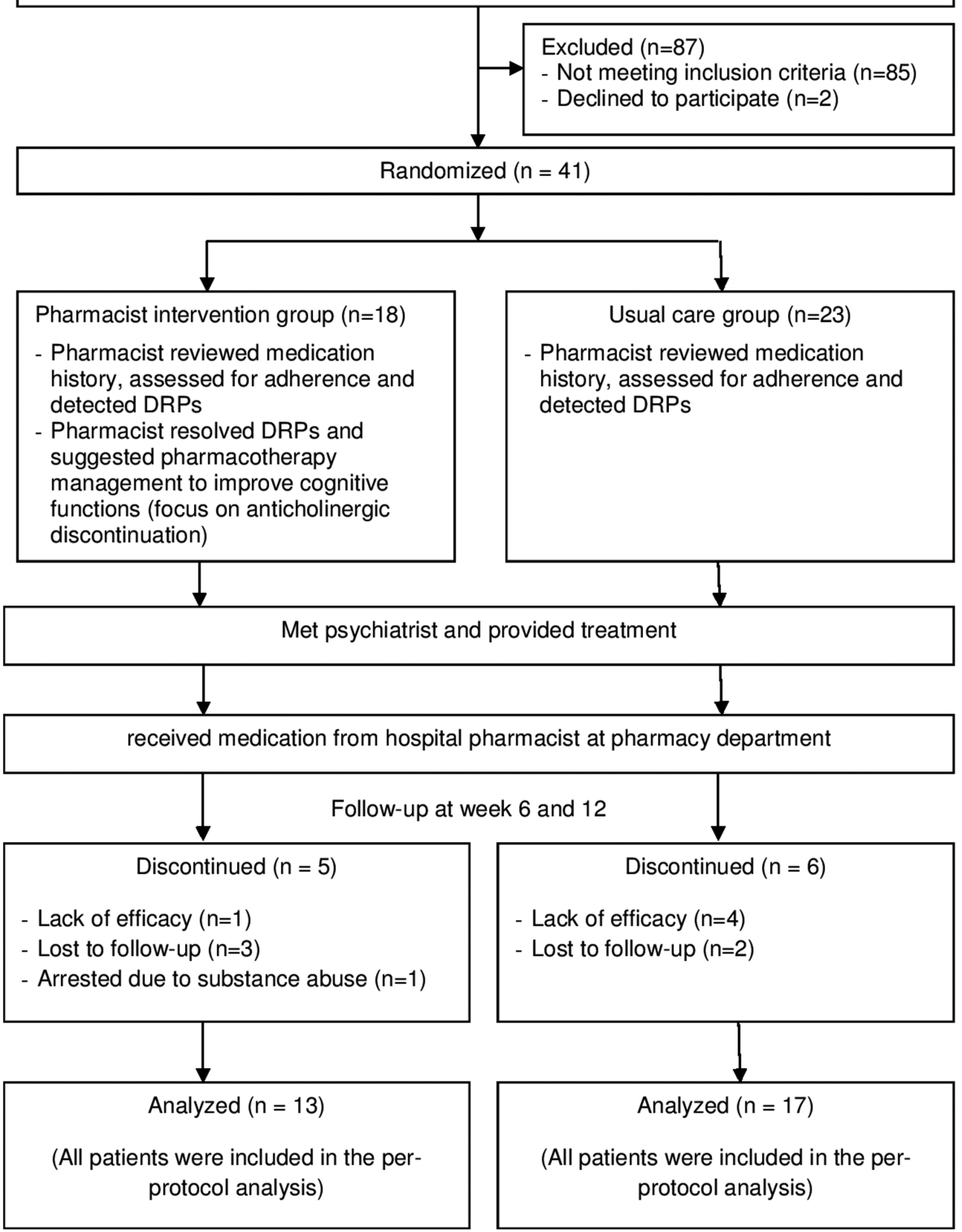

Figure I Flow diagram of patients through the study protocol (according to CONSORT 2010 statement). Abbreviation: DRPs, drug-related problems. 
Table I The cognitive tests used to assess the three cognitive domains in the study

\begin{tabular}{|c|c|c|c|}
\hline \multirow[t]{2}{*}{ Cognitive tests } & \multicolumn{3}{|l|}{ Cognitive domains } \\
\hline & Verbal learning and memory & Executive function & Speed of processing \\
\hline WMS & $\sqrt{ }$ & & \\
\hline WCST & & $\sqrt{ }$ & \\
\hline Stroop Color and Word Test & & $\sqrt{ }$ & \\
\hline TMT A & & & $\sqrt{ }$ \\
\hline TMT B & & $\sqrt{ }$ & \\
\hline
\end{tabular}

Abbreviations: TMT, Trail Making Test; WCST, Wisconsin Card Sorting Test; WMS, Wechsler Memory Scale.

applied for the number of DRPs. All analyses were carried out using the SPSS version 21.

\section{Results}

\section{Patient characteristics}

In total, 41 patients were enrolled and 30 patients completed the study period (Figure 1). Of whom, 13 and 17 patients were in the pharmacist intervention and usual care groups, respectively. Demographic and clinical characteristics of these patients are shown in Table 2. There were no significant differences among the treatment groups for most characteristics, except that patients in the usual care group had a higher baseline unemployment $(P=0.023)$. However, there was no correlation between unemployment rate and WCST perseverative errors $(r=-0.316, P=0.293)$.

\section{Cognitive outcomes}

Baseline and end point scores of the cognitive tests of the pharmacist intervention and usual care groups are given in Table 3. Comparing within the pharmacist intervention group at the end of study, WCST perseverative errors significantly improved from baseline at $21.23 \pm 11.81$ to $9.61 \pm 6.47$ at week $12(P=0.003$; Table 3). Other cognitive tests that showed a significant improvement from baseline were TMT B, WMS trial 1, WMS total words recalled, and short delay free recall trial. Focusing within the usual care group, there were significant improvements in WCST number of categories completed, WMS trial 1, WMS total words recalled, and short delay free recall trial from baseline to week 12, but not for WCST perseverative errors.

Comparison between groups at week 12 revealed that WCST perseverative errors was the only test that showed a more significant improvement in the pharmacist intervention group than that in the usual care group ( $\triangle$ WCST perseverative errors: $-11.62 \pm 12.78$ and $-2.14 \pm 6.96$, respectively; $P=0.017$ ).

\section{Psychopathological outcomes}

At the end of study, BPRS scores were significantly lower in the pharmacist intervention group than those in the usual care group ( $36.08 \pm 3.62$ to $24.69 \pm 2.72$ and $38.29 \pm 6.38$ to $36.35 \pm 7.71$, respectively; $P<0.001$; Figure 2). Regarding treatment response, $61.54 \%$ in the pharmacist intervention group achieved response, while no responders did so in the usual care group.

\section{DRPs}

At baseline, the numbers of DRPs were 27 and 41 in the pharmacist intervention and usual care groups (2.08 and 2.41 DRPs per patient), respectively (Table 4). DRPs mostly found among both groups were unnecessary drug therapy, especially using anticholinergics for prevention of EPS. At baseline, there were $92.31 \%$ (12 of 13) and $100 \%$ (17 of 17) of patients in the pharmacist intervention and usual care groups who received anticholinergic therapy. Of these patients, 12 and 15 patients in the pharmacist intervention and usual care groups qualified for consideration of anticholinergic discontinuation as assessed by the clinical pharmacist followed by the study criteria (no EPS at least 4 weeks and no history of serious EPS). Adverse drug reactions (ADRs) such as weight gain, EPS, and hyperprolactinemia were other DRPs found in both groups. At the end of the study, the number of identified DRPs in the pharmacist intervention and usual care groups was 4 and 37, which was a decrease from baseline of $85.19 \%$ and $9.76 \%$, respectively.

Regarding interventions throughout the study, there were 28 interventions in the pharmacist intervention group by the clinical pharmacist (acceptance rate was $92.86 \%$ of these interventions), while there were only four interventions in the usual care group by the psychiatrists. In the pharmacist intervention group, unnecessary drug therapy relating to preventing EPS with anticholinergic therapy was the highest intervention (12 interventions), of which all were accepted by the psychiatrists. Anticholinergic agents were gradually decreased and discontinued for 10 patients. Anticholinergic agents could not be discontinued for two patients, in which one of them was under the tapering schedule of anticholinergics and unable to stop before the end of study, while the 
Table 2 Baseline characteristics of the study participants

\begin{tabular}{|c|c|c|}
\hline Characteristics & Pharmacist intervention group $(n=13)$ & Usual care group $(n=17)$ \\
\hline Mean age, years (SD) & $38.31(4.96)$ & $36.06(9.88)$ \\
\hline Sex (\% female) & 69.2 & 58.8 \\
\hline Mean body mass index, $\mathrm{kg} / \mathrm{m}^{2}(\mathrm{SD})$ & $25.21(5.87)$ & $23.23(5.4)$ \\
\hline Education, years (SD) & $14.54(4.16)$ & $13(4.4)$ \\
\hline \multicolumn{3}{|l|}{ Substance use (\%) } \\
\hline - Smoking & 30.8 & 41.2 \\
\hline - Alcohol & 23.1 & 17.6 \\
\hline - Amphetamine & 15.4 & 17.6 \\
\hline - Cannabis & 0 & 11.8 \\
\hline \multicolumn{3}{|l|}{ Comorbid psychiatric disorders (\%) } \\
\hline - Depressive disorder & 7.69 & 11.76 \\
\hline - Bipolar disorder & 7.69 & 5.88 \\
\hline - Attention deficit hyperactivity disorder & 7.69 & 0 \\
\hline Unemployment (\%) ${ }^{\mathrm{a}}$ & 38.5 & 82.4 \\
\hline Age at onset of illness, years (SD) & $32.89(6.42)$ & $30.99(10.11)$ \\
\hline Duration of illness, years (SD) & $5.42(3.35)$ & $5.07(3.03)$ \\
\hline Number of prior hospitalizations (SD) & $\mathrm{I}(\mathrm{I} .2)$ & $0.82(1.59)$ \\
\hline \multicolumn{3}{|l|}{ Current antipsychotic use (\%) } \\
\hline - $F G A s^{b}$ & 46.15 & 58.82 \\
\hline - $S G A s^{c}$ & 53.85 & 64.71 \\
\hline Anticholinergic use (\%) & 92.31 & 100 \\
\hline Adherence (\%) ${ }^{\mathrm{d}}$ & 84.78 & 85.79 \\
\hline BPRS, mean (SD) & $36.08(3.62)$ & $38.29(6.38)$ \\
\hline \multicolumn{3}{|l|}{ WCST, mean (SD) } \\
\hline - Number of categories completed & $1.46(1.45)$ & $\mathrm{I} .12(\mathrm{I} .4 \mathrm{I})$ \\
\hline - Perseverative errors & $21.23(11.8 I)$ & $13.65(10.5)$ \\
\hline \multicolumn{3}{|l|}{ WMS, mean (SD) } \\
\hline - Trial I & $3.69(1.75)$ & $3.59(2.03)$ \\
\hline - Total words recalled (trials I-4) & $22.84(7.54)$ & $20.29(7.56)$ \\
\hline - Short delay free recall trial & $5(2.2)$ & $4.59(2.27)$ \\
\hline Stroop Color and Word Test, mean (SD) & $30.77(\mathrm{II} .1 \mathrm{I})$ & $28.94(8.74)$ \\
\hline TMT A, mean $(S D)$ & $50.54(25.5 \mathrm{I})$ & $62.12(40.96)$ \\
\hline TMT B, mean (SD) & $165.38(90.41)$ & I52.53 (77.48) \\
\hline
\end{tabular}

Notes: ${ }^{\mathrm{a}}$-value $<0.05$. ${ }^{\mathrm{b} F G A s}$ including haloperidol ( $0 \%$ in the pharmacist intervention group, $3.68 \%$ in the usual care group), flupentixol ( $0 \%$ in the pharmacist intervention group, $3.68 \%$ in the usual care group), trifluoperazine ( $7.6 \% \%$ in the pharmacist intervention group, $0 \%$ in the usual care group), perphenazine (38.46\% in the pharmacist intervention group, $25.73 \%$ in the usual care group), chlorpromazine ( $0 \%$ in the pharmacist intervention group, $14.7 \%$ in the usual care group), fluphenazine decanoate ( $0 \%$ in the pharmacist intervention group, $7.35 \%$ in the usual care group), haloperidol decanoate ( $0 \%$ in the pharmacist intervention group, $3.68 \%$ in the usual care group). ' $5 G A s$ including risperidone (38.47\% in the pharmacist intervention group, $52.93 \%$ in the usual care group), olanzapine (15.38\% in the pharmacist intervention group, $5.89 \%$ in the usual care group), paliperidone ( $0 \%$ in the pharmacist intervention group, $5.89 \%$ in the usual care group). ${ }^{d}$ Adherence is defined as following at least $80 \%$ of the medication regimen.

Abbreviations: BPRS, Brief Psychiatric Rating Scale; FGAs, first-generation antipsychotics; SGAs, second-generation antipsychotics; TMT, Trail Making Test; WCST, Wisconsin Card Sorting Test; WMS, Wechsler Memory Scale.

Table 3 Cognitive outcomes in pharmacist intervention and usual care groups at baseline and week 12

\begin{tabular}{|c|c|c|c|c|c|c|c|}
\hline \multirow[t]{2}{*}{ Cognitive tests } & \multicolumn{3}{|c|}{ Pharmacist intervention group $(n=13)$} & \multicolumn{3}{|c|}{ Usual care group $(n=17)$} & \multirow[t]{2}{*}{$P$-value } \\
\hline & $\begin{array}{l}\text { Baseline } \\
\text { (mean } \pm S D)\end{array}$ & $\begin{array}{l}\text { Week I2 } \\
\text { (mean } \pm \text { SD) }\end{array}$ & P-value ${ }^{a}$ & $\begin{array}{l}\text { Baseline } \\
\text { (mean } \pm S D)\end{array}$ & $\begin{array}{l}\text { Week I2 } \\
(\text { mean } \pm S D)\end{array}$ & $P$-value ${ }^{b}$ & \\
\hline \multicolumn{8}{|l|}{ WCST } \\
\hline - Number of categories complete & $1.46 \pm 1.45$ & $2.15 \pm 1.86$ & 0.124 & $1.12 \pm 1.4 \mid$ & $1.7 \mathrm{I} \pm \mathrm{I} .6 \mathrm{I}$ & $0.024^{d}$ & 0.742 \\
\hline - Perseverative errors & $21.23 \pm 11.81$ & $9.61 \pm 6.47$ & $0.003^{d}$ & $13.65 \pm 10.5$ & $\mid 1 \pm 7.51$ & 0.187 & $0.017^{\mathrm{d}}$ \\
\hline \multicolumn{8}{|l|}{ WMS (word lists subtest) } \\
\hline - Trial I & $3.69 \pm 1.75$ & $5.69 \pm 2.06$ & $0.005^{d}$ & $3.59 \pm 2.03$ & $4.82 \pm 2.35$ & $0.015^{d}$ & 0.281 \\
\hline - Total words recalled & $22.85 \pm 7.54$ & $31 \pm 5.64$ & $0.002^{\mathrm{d}}$ & $20.29 \pm 7.56$ & $24.12 \pm 8.51$ & $0.016^{\mathrm{d}}$ & 0.059 \\
\hline - Short delay free recall trial & $5 \pm 2.2$ & $6.31 \pm 3.01$ & $0.048^{d}$ & $4.59 \pm 2.27$ & $6.24 \pm 2.33$ & $0.009^{d}$ & 0.773 \\
\hline Stroop Color and Word Test $(>25)$ & $30.77 \pm 11.11$ & $33.38 \pm 12.43$ & 0.272 & $28.94 \pm 8.74$ & $28.12 \pm 9.62$ & 0.521 & 0.341 \\
\hline TMT A (3I-39) & $50.54 \pm 25.5 \mathrm{I}$ & $41.92 \pm 22.81$ & 0.196 & $62.12 \pm 40.96$ & $54 \pm 33.6 \mathrm{I}$ & 0.142 & 0.650 \\
\hline TMT B (75-92) & $|65.38 \pm 90.4|$ & $|26.3| \pm 83.25$ & $0.015^{d}$ & $152.53 \pm 77.48$ & $|58.4| \pm 100.91$ & 0.918 & 0.079 \\
\hline
\end{tabular}

Notes: ${ }^{a} P$-value of cognitive tests between baseline and end point within the pharmacist intervention group. ${ }^{b} P$-value of cognitive tests between baseline and end point within the usual care group. ${ }^{C} P$-value of cognitive tests between the pharmacist intervention group and the usual care group at baseline and end point. ${ }^{d} P$-value $<0.05$. Abbreviations: TMT, Trial Making Test; WCST, Wisconsin Card Sorting Test; WMS, Wechsler Memory Scale. 
other developed mild EPS during the tapering schedule. In the usual care group, all patients continued using anticholinergics throughout the study.

\section{Discussion}

To the best of our knowledge, this was the first prospective study aiming to determine the effectiveness of pharmacist intervention focusing on anticholinergic discontinuation in patients with schizophrenia with the emphasis on cognitive outcomes. Findings of this study demonstrate the pharmacist's role in the multidisciplinary team for cognitive and psychopathologic improvements in patients with schizophrenia.

The duration of study, the time to reevaluate the changes in cognitive functions, was 12 weeks. This was sufficient for detecting an improvement in WCST perseverative errors after

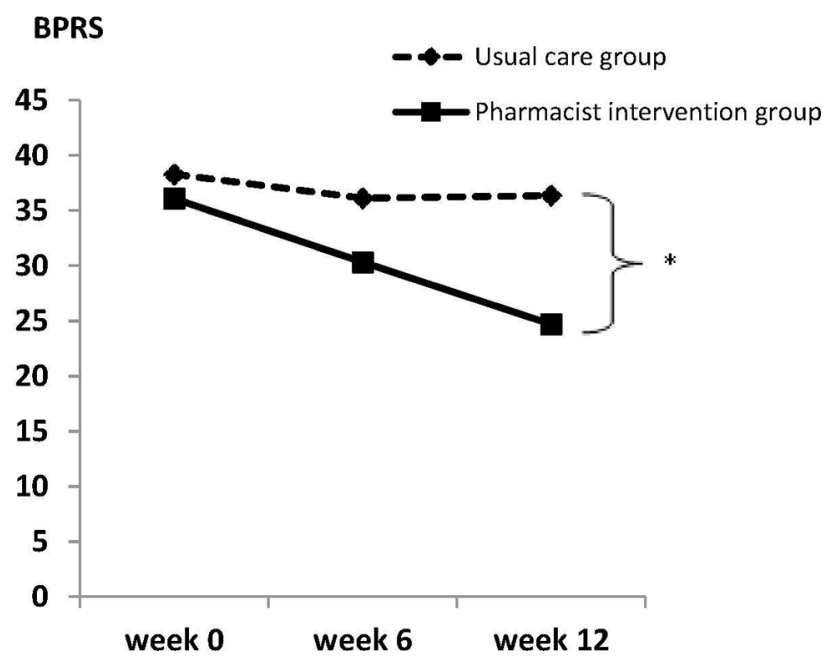

Figure 2 Comparison of BPRS between pharmacist intervention and usual care groups (*P-value $<0.05)$.

Abbreviation: BPRS, Brief Psychiatric Rating Scale. any pharmacological interventions had been received because cognitive and neuroanatomical changes could occur within 12 weeks. ${ }^{16,30-32}$ In our primary outcome analysis, WCST perseverative errors at the end of study in the pharmacist intervention group significantly improved from baseline and significantly differed from the usual care group. These results might be related to pharmacist intervention for the discontinuation of anticholinergic drugs. Anticholinergic agents are drugs that block the action of acetylcholine via inhibiting postsynaptic muscarinic $\mathrm{M}_{1}$ receptor. This receptor is highly concentrated at prefrontal cortex and hippocampus, which relates to action of executive function and memory, respectively. In addition, the evidence revealed that $M_{1}$ receptor expression had decreased in the prefrontal cortex for patients with schizophrenia. ${ }^{33}$ Therefore, using anticholinergic drugs over the long term could cause executive dysfunction. Previous studies showed that gradual discontinuation of anticholinergics helped to improve cognitive functions and patient was safe from the recurrence of EPS. ${ }^{19,20}$ As most patients in the study were on anticholinergics (only one was not on anticholinergics in the pharmacist intervention group), so discontinuation of anticholinergics was the key pharmacological intervention to improve executive function in this study. In addition, the results, excluding the one patient not on anticholinergics, also found significant improvement in WCST perseverative errors from baseline to end point within the pharmacist intervention group $(22.67 \pm 11.24$ to $10 \pm 6.61$; $P=0.002$ ). Patients in the usual care group continued using anticholinergics throughout the study despite experiencing no EPS. Hence, there was no significant improvement in WCST perseverative errors at the end of the study in the usual care group. In addition to the discontinuation of anticholinergics, improvement of executive function in the

Table 4 Comparison of DRPs between pharmacist intervention and usual care groups

\begin{tabular}{|c|c|c|c|c|}
\hline \multirow[t]{2}{*}{ DRPs } & \multicolumn{2}{|c|}{ Pharmacist intervention group $(n=13)$} & \multicolumn{2}{|c|}{ Usual care group $(n=\mid 7)$} \\
\hline & Baseline & Week 12 & Baseline & Week 12 \\
\hline Need additional drug therapy & 0 & 0 & I & I \\
\hline Unnecessary drug therapy & 14 & 0 & 23 & 22 \\
\hline $\begin{array}{l}\text { - Treating avoidable ADR } \\
\text { (use of anticholinergics) }\end{array}$ & 12 & 0 & 15 & 15 \\
\hline - Duplicate drug therapy & 0 & 0 & 5 & 5 \\
\hline - No medical indication & 2 & 0 & 3 & 2 \\
\hline Wrong drug & 2 & 1 & I & 1 \\
\hline Dosage too low & 1 & 0 & I & 1 \\
\hline Dosage too high & 0 & 0 & 0 & 0 \\
\hline ADR & 8 & 3 & 11 & 10 \\
\hline Adherence problem & 2 & 0 & 4 & 2 \\
\hline Total DRPs & 27 & 4 & 41 & 37 \\
\hline
\end{tabular}

Abbreviations: ADR, adverse drug reaction; DRPs, drug-related problems. 
pharmacist intervention group might be related to the better psychopathological outcome (BPRS) than that in the usual care group. Berman et $\mathrm{al}^{34}$ reported that executive function was highly correlated with negative subscores of Positive and Negative Syndrome Scale. This phenomenon might relate to hypodopaminergic state at prefrontal cortex, which shared pathophysiology between executive dysfunction and negative symptoms in schizophrenia. Therefore, effective treatment of negative symptoms could promote cognitive functions. ${ }^{21}$ Treatment strategies to improve negative symptoms were based on limited evidence-based pharmacotherapy such as adding on antidepressants or substituting SGAs for FGAs. ${ }^{22-26}$ Regarding the pharmacist intervention group, there were two interventions to optimize the pharmacological treatment of negative symptoms. However, only one was accepted, which was adding antidepressant for a patient with prominent negative symptoms. After 12 weeks, WCST perseverative errors of this patient decreased from 22 to 7 ; likewise, an improvement was observed in negative symptoms.

Concerning the other cognitive outcomes, TMT B was another test for executive function that also showed a significant improvement from baseline for the pharmacist intervention group, while no significant improvement was recorded for the usual care group. This result helped confirm the effect of pharmacist intervention on executive function in patients with schizophrenia. For TMT A, it measures processing speed primarily and has a high sensitivity to adverse effects of medication on motor system such as EPS and sedation. ${ }^{35}$ Therefore, managing these adverse events could help to improve TMT A scores. Nevertheless, our participants experienced a few of these adverse effects; thus, this might be a cause of the insignificant improvement on TMT A for both groups. Focusing on WMS, this test was used to examine verbal learning and memory domain. Our results found that there was a significant improvement of WMS from baseline to the end of study for both groups with no significant difference between groups. These might be related to its high sensitivity for improvement and that it could be improved easier than other cognitive domains. ${ }^{36-39}$

Some limitations of our study should be commented on. First, a high number of patients withdrew early, which was more than anticipated. Most of them were lost to follow-up. This might be because our missing patients lived in regional areas and possibly had limitations concerning finance. Second, our study was an open-label study design. However, the study blinded the psychologists who assessed the cognitive functions at baseline and 12 weeks, which should have no effect on primary outcome. Third, not all pharmacist interventions could be implemented in this study such as substituting SGAs for FGAs in patients who had executive function impairment based on cognitive function tests. This was related to the impoverished economic status of our patients and several SGAs not being listed on the National List of Essential Medicines in Thailand. Therefore, the main pharmacist intervention in this study was anticholinergic discontinuation. Apart from this, further studies with large sample sizes and long-term follow-up to determine the impact of pharmacist intervention in patients with schizophrenia should be conducted.

\section{Conclusion}

This study first demonstrated that pharmacist intervention together with psychiatrist could help to better improve cognitive functions, especially executive function and psychopathological symptoms, in outpatients with schizophrenia than those in usual care. These beneficial effects might be caused by delivering pharmaceutical care process, which played an important role in decreasing number of DRPs that commonly occur in patients with schizophrenia. Unnecessary drug therapy emphasizing the receiving of anticholinergics for prevention of EPS was the most common problem. Hence, therapeutic indications for anticholinergic therapy should be clarified for all patients with schizophrenia and discontinuation should be commenced if there is no indication. Our results may be useful to build up and develop the role of the pharmacist in caring for patients with schizophrenia.

\section{Acknowledgments}

We wish to acknowledge physicians who contributed to this research, Dr Norrawee Phoomchan, Dr Pholphat Losatiankij, and Dr Charnwit Skawatananon. We also acknowledge the helpful consultation from a native speaker who is a medical English specialist, Mr Stephen Pinder, and Mr Stephen Lorrinan who proofread our full manuscript. We also acknowledge all the patients who participated in the trial. This work was supported by Siam University, Thailand. The funder played no involvement in study design, collection of data, analysis, interpretation, and writing of the report.

\section{Author contributions}

Sathienluckana $\mathrm{T}$ conducted the protocol of research, contributed to data collection, analyzed the data, and drafted and critically reviewed the manuscript. Unaharassamee $\mathrm{W}$ contributed to data collection and critically reviewed the manuscript. Suthisisang C provided ideas, contributed to the design of the research, and critically reviewed the manuscript. 
Suanchang O contributed to data collection, contacted physicians and other healthcare professionals to join the research, and critically reviewed the manuscript. Suansanae $T$ provided ideas and contributed to the design of the research, assisted in analyzing the data and drafting the manuscript, and critically reviewed the manuscript. All authors contributed toward data analysis, drafting and critically revising the paper and agree to be accountable for all aspects of the work.

\section{Disclosure}

The authors report no conflicts of interest in this work.

\section{References}

1. Schulz SC, Murray A. Assessing cognitive impairment in patients with schizophrenia. J Clin Psychiatry. 2016;77(Suppl 2):3-7.

2. Keefe RS. The longitudinal course of cognitive impairment in schizophrenia: an examination of data from premorbid through posttreatment phases of illness. J Clin Psychiatry. 2014;75(Suppl 2):8-13.

3. Lieberman JA, Perkins D, Belger A, et al. The early stages of schizophrenia: speculations on pathogenesis, pathophysiology, and therapeutic approaches. Biol Psychiatry. 2001;50(11):884-897.

4. Green MF. Cognitive impairment and functional outcome in schizophrenia and bipolar disorder. J Clin Psychiatry. 2006;67(10):e12-e17.

5. Green MF, Kern RS, Heaton RK. Longitudinal studies of cognition and functional outcome in schizophrenia: implications for MATRICS. Schizophr Res. 2004;72(1):41-51.

6. Green MF, Kern RS, Braff DL, Mintz J. Neurocognitive deficits and functional outcome in schizophrenia: are we measuring the "right stuff"? Schizophr Bull. 2000;26(1):119-136.

7. Chen EY, Hui CL, Dunn EL, et al. A prospective 3-year longitudinal study of cognitive predictors of relapse in first-episode schizophrenic patients. Schizophr Res. 2005;77(1):99-104.

8. Vinogradov S, Schulz SC. Behavioral and emerging pharmacologic treatment options for cognitive impairment in schizophrenia. J Clin Psychiatry. 2016;77(Suppl 2):12-16.

9. Dempsey JT, Matta LS, Carter DM, et al. Assessment of Drug TherapyRelated Issues in an Outpatient Heart Failure Population and the Potential Impact of Pharmacist-Driven Intervention. $J$ Pharm Pract. 2017;30(3):318-323.

10. Schultz JL, Horner KE, McDanel DL, et al. Comparing clinical outcomes of a pharmacist-managed diabetes clinic to usual physician-based care. J Pharm Pract. 2018;31(3):268-271.

11. Robinson JD, Segal R, Lopez LM, Doty RE. Impact of a pharmaceutical care intervention on blood pressure control in a chain pharmacy practice. Ann Pharmacother. 2010;44(1):88-96.

12. Rubio-Valera M, Serrano-Blanco A, Magdalena-Belío J, et al. Effectiveness of pharmacist care in the improvement of adherence to antidepressants: a systematic review and meta-analysis. Ann Pharmacother. 2011;45(1):39-48.

13. Wongpakaran R, Suansanae T, Tan-Khum T, Kraivichian C, Ongarjsakulman R, Suthisisang C. Impact of providing psychiatry specialty pharmacist intervention on reducing drug-related problems among children with autism spectrum disorder related to disruptive behavioural symptoms: A prospective randomized open-label study. J Clin Pharm Ther. 2017;42(3):329-336.

14. Canales PL, Dorson PG, Crismon ML. Outcomes assessment of clinical pharmacy services in a psychiatric inpatient setting. Am J Health Syst Pharm. 2001;58(14):1309-1316.

15. Ogino S, Miyamoto S, Miyake N, Yamaguchi N. Benefits and limits of anticholinergic use in schizophrenia: focusing on its effect on cognitive function. Psychiatry Clin Neurosci. 2014;68(1):37-49.
16. Purdon SE, Jones BD, Stip E, et al. Neuropsychological change in early phase schizophrenia during 12 months of treatment with olanzapine, risperidone, or haloperidol. The Canadian Collaborative Group for research in schizophrenia. Arch Gen Psychiatry. 2000;57(3):249-258.

17. Gold S, Arndt S, Nopoulos P, O'Leary DS, Andreasen NC. Longitudinal study of cognitive function in first-episode and recent-onset schizophrenia. Am J Psychiatry. 1999;156(9):1342-1348.

18. Harvey PD, Rabinowitz J, Eerdekens M, Davidson M. Treatment of cognitive impairment in early psychosis: a comparison of risperidone and haloperidol in a large long-term trial. Am J Psychiatry. 2005;162(10):1888-1895.

19. Ogino S, Miyamoto S, Tenjin T, et al. Effects of discontinuation of longterm biperiden use on cognitive function and quality of life in schizophrenia. Prog Neuropsychopharmacol Biol Psychiatry. 2011;35(1): 78-83.

20. Mori K, Yamashita H, Nagao M, Horiguchi J, Yamawaki S. Effects of anticholinergic drug withdrawal on memory, regional cerebral blood flow and extrapyramidal side effects in schizophrenic patients. Pharmacopsychiatry. 2002;35(1):6-11.

21. Harvey PD, Koren D, Reichenberg A, Bowie CR. Negative symptoms and cognitive deficits: what is the nature of their relationship? Schizophr Bull. 2006;32(2):250-258.

22. Möller HJ. Management of the negative symptoms of schizophrenia: new treatment options. CNS Drugs. 2003;17(11):793-823.

23. Silver H. Selective serotonin reuptake inhibitor augmentation in the treatment of negative symptoms of schizophrenia. Int Clin Psychopharmacol. 2003;18(6):305-313.

24. Singh SP, Singh V, Kar N, Chan K. Efficacy of antidepressants in treating the negative symptoms of chronic schizophrenia: meta-analysis. $\mathrm{Br} \mathrm{J}$ Psychiatry. 2010;197(3):174-179.

25. Zoccali R, Muscatello MR, Cedro C, et al. The effect of mirtazapine augmentation of clozapine in the treatment of negative symptoms of schizophrenia: a double-blind, placebo-controlled study. Int Clin Psychopharmacol. 2004;19(2):71-76.

26. Berk M, Ichim C, Brook S. Efficacy of mirtazapine add on therapy to haloperidol in the treatment of the negative symptoms of schizophrenia: a double-blind randomized placebo-controlled study. Int Clin Psychopharmacol. 2001;16(2):87-92.

27. Keefe RS, Young CA, Rock SL, et al; HGGN Study Group. One-year double-blind study of the neurocognitive efficacy of olanzapine, risperidone, and haloperidol in schizophrenia. Schizophr Res. 2006;81(1):1-15.

28. Sakurai H, Bies RR, Stroup ST, et al. Dopamine D2 receptor occupancy and cognition in schizophrenia: analysis of the CATIE data. Schizophr Bull. 2013;39(3):564-574.

29. van Mil JW, Westerlund LO, Hersberger KE, Schaefer MA. Drug-related problem classification systems. Ann Pharmacother. 2004;38(5):859-867.

30. Riedel M, Müller N, Spellmann I, et al. Efficacy of olanzapine versus quetiapine on cognitive dysfunctions in patients with an acute episode of schizophrenia. Eur Arch Psychiatry Clin Neurosci. 2007;257(7):402-412.

31. Riedel M, Spellmann I, Strassnig M, et al. Effects of risperidone and quetiapine on cognition in patients with schizophrenia and predominantly negative symptoms. Eur Arch Psychiatry Clin Neurosci. 2007;257(6):360-370.

32. Lieberman JA, Tollefson GD, Charles C, et al; HGDH Study Group. Antipsychotic drug effects on brain morphology in first-episode psychosis. Arch Gen Psychiatry. 2005;62(4):361-370.

33. Dean B, Mcleod M, Keriakous D, Mckenzie J, Scarr E. Decreased muscarinic1 receptors in the dorsolateral prefrontal cortex of subjects with schizophrenia. Mol Psychiatry. 2002;7(10):1083-1091.

34. Berman I, Viegner B, Merson A, Allan E, Pappas D, Green AI. Differential relationships between positive and negative symptoms and neuropsychological deficits in schizophrenia. Schizophr Res. 1997;25(1):1-10.

35. Keefe RS, Eesley CE. Neurocognition in schizophrenia. In: Sadock BJ, Sadock VA, Ruiz P, editors. Kaplan \& Sadock's: Comprehensive Textbook of Psychiatry. 9th ed. New York: Wolters Kluwer; 2009:1531-1540. 
36. Prentice KJ, Gold JM, Buchanan RW. The Wisconsin Card Sorting impairment in schizophrenia is evident in the first four trials. Schizophr Res. 2008;106(1):81-87.

37. Monchi O, Petrides M, Petre V, Worsley K, Dagher A. Wisconsin Card Sorting revisited: distinct neural circuits participating in different stages of the task identified by event-related functional magnetic resonance imaging. J Neurosci. 2001;21(19):7733-7741.
38. Harvey PD, Green MF, Mcgurk SR, Meltzer HY. Changes in cognitive functioning with risperidone and olanzapine treatment: a largescale, double-blind, randomized study. Psychopharmacology (Berl). 2003;169(3-4):404-411.

39. Bervoets C, Morrens M, Vansteelandt K, et al. Effect of aripiprazole on verbal memory and fluency in schizophrenic patients: results from the ESCAPE study. CNS Drugs. 2012;26(11):975-982. 


\section{Supplementary material}

Table SI The cognitive tests used to assess the three cognitive domains in the study

\begin{tabular}{|c|c|c|}
\hline Cognitive domain & Neurocognitive test & Description \\
\hline Executive function & $\begin{array}{l}\text { Stroop Color and } \\
\text { Word Test } \\
\text { TMT B }\end{array}$ & $\begin{array}{l}\text { - The test was a complex problem-solving task that probably requires multiple cognitive } \\
\text { processes rather than a single unitary function. The subject was asked to match test } \\
\text { cards to reference cards according to the color, shape, or number of stimuli on the } \\
\text { cards. After a fixed number of correct matches, the sorting rule was changed without } \\
\text { notice, and the subject was shifted to a new mode of classification. The test measured } \\
\text { executive function that was correlated with dorsolateral prefrontal cortex. Score of } \\
\text { WCST used I) number of category complete that was an indicator for categorizing } \\
\text { ability (incremental score means improvement ranging from } 0 \text { to a maximum of } 6 \text { ) and 2) } \\
\text { perseverative errors (number of errors that subject was incorrect in matching test cards } \\
\text { because used the same rule as the previous mode, so incremental score means worse } \\
\text { cognitive symptoms. Score ranged from } 0 \text { to a maximum of } 64 \text { ) } \\
\text { - The test measures ability to name the color of a series of colored words rather than } \\
\text { read the color names. In normal population, score should be } \geq 25 \text { words, and incremental } \\
\text { score means a cognitive improvement } \\
\text { - The test can provide information about visual search speed, scanning, speed of } \\
\text { processing, mental flexibility, as well as executive functioning, which is sensitive to } \\
\text { prefrontal cortex function. The test measures the time required to draw lines connecting } \\
\text { circles by shifting from numbers to letters (normal range was about } 75-92 \text { seconds, and } \\
\text { score reduction means executive function improvement) }\end{array}$ \\
\hline $\begin{array}{l}\text { Verbal learning and } \\
\text { memory }\end{array}$ & $\begin{array}{l}\text { WMS: word lists } \\
\text { subtest }\end{array}$ & $\begin{array}{l}\text { - The word lists subtest consists of } 12 \text { unrelated words (List A) presented in four learning } \\
\text { trials, with recall measured after each presentation. A distracter list of I } 2 \text { different words } \\
\text { (List B) is then administered, followed immediately with a short delay free recall trial } \\
\text { of the original list. Score of WMS used I) trial I (word recall in trial I); 2) total word } \\
\text { recall (total word recall in List A for four times); and 3) short delay free recall (word } \\
\text { recall of list A immediately after test in List B). Increased score of all these tests means } \\
\text { improvement in learning and memory function }\end{array}$ \\
\hline Speed of processing & TMT A & $\begin{array}{l}\text { - Time required to complete the test that assessed motor speed and attention (normal } \\
\text { range was about } 31-39 \text { seconds, and score reduction means improvement in processing } \\
\text { speed) }\end{array}$ \\
\hline
\end{tabular}

Abbreviations: TMT, Trail Making Test; WCST, Wisconsin Card Sorting Test; WMS, Wechsler Memory Scale.

Integrated Pharmacy Research and Practice is an international, peer-reviewed, open access, online journal, publishing original research, reports, reviews and commentaries on all areas of academic and professional pharmacy practice. This journal aims to represent the academic output of pharmacists and pharmacy practice with particular focus on integrated care. All papers are carefully peer reviewed to ensure the highest standards as well as ensuring that we are informing and stimulating pharmaceutical professionals. The manuscript management system is completely online and includes a very quick and fair peer-review system, which is all easy to use. Visit http://www.dovepress.com/ testimonials.php to read real quotes from published authors. 